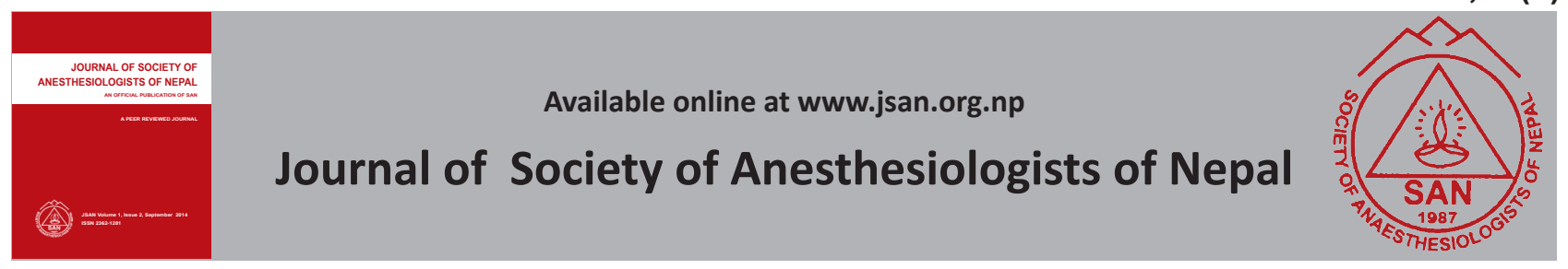

Case Report

\title{
Valproic acid induced acute necrotizing pancreatitis
}

\author{
Rabindra Gurung*, Asish Subedi**, Balkrishna Bhattarai** \\ *KIST Medical College Teaching Hospital, \\ **BP Koirala Institute of Health and Sciences, Dharan, Nepal
}

\author{
ARTICLE INFO \\ Article history \\ Received 28.07.2014 \\ Accepted 27.08.2014 \\ Published 08.09.2014 \\ (C) Authors retain copyright \\ and grant the journal right \\ of first publication with the \\ work simultaneously licensed \\ under a Creative Commons \\ Attribution License that allows \\ others to share the work with \\ an acknowledgment of the \\ work's authorship and initial \\ publication in this journal.
}

\begin{abstract}
Background: Valproic acid induced pancreatitis is a rare entity with an estimated incidence of one in 40000. Occurrence of acute necrotizing pancreatitis is even more uncommon with only very few cases being reported till date. We present a case of valproic acid associated acute necrotizing pancreatitis that complicated to develop pancreatic pseudo cyst.
\end{abstract}

Case discussion: A 21-year-old male presented in our emergency room with sudden onset of severe epigastric pain along with nausea and vomiting. He gave history of having on valproic acid therapy for the last 13 months for seizure disorder. There was no history suggestive of gall stone disease, alcohol intake, hyperlipidemia, abdominal trauma or any surgical intervention. Clinical suspicion of acute pancreatitis was confirmed by revealing of necrotizing pancreatitis in contrast enhanced computerized tomography. Patient was admitted in the intensive care unit where valproic acid was stopped and phenytoin started. Clinical improvement was observed with conservative management while mild intermittent abdominal pain, nausea and vomiting persisted. Repeat computerized tomography done later revealed features of pancreatic pseudo cyst for which patient underwent definitive surgical management.

Conclusions: Today, valproic acid is widely used for multiple disease conditions. Starting from 1979 till 2010, 107 cases of valproic acid induced acute pancreatitis have been reported. Acute pancreatitis is a serious condition with significant mortality despite optimal treatment. Unfortunately, it is underreported and underestimated especially following valproic acid use. Increasing frequency of valproic acid associated pancreatitis demands reconsideration of its use.

Key-words: Acute necrotizing Pancreatitis, adverse effects, valproic acid.

How to cite this article: Gurung R, Subedi A, Bhattarai B. Valproic acid induced acute necrotizing pancreatitis. JSAN 2014;1:86-88

\section{Introduction}

Valproic acid (VPA) is a widely used anti epileptic drug. However, its use has been related to certain uncommon but serious adverse effects such as liver dysfunction, coagulopathy, encephalopathy and pancreatitis. VPA induced pancreatitis is a rare entity with an estimated incidence of one in 40000. Starting from 1979 to 2010, 107 cases of VPA induced acute pancreatitis (AP) have been reported. ${ }^{1}$ Occurrence of acute necrotizing pancreatitis

Correspondence: Rabindra Gurung

Department of Anaesthesia and Critical care, KIST Medical College Teaching Hospital, Imadol, Lalitpur, Nepal

Email: gurung_rabindra@yahoo.com 
is even more uncommon with only very few cases being reported to date. Here, we present a case of VPA associated acute necrotizing pancreatitis that complicated to develop pancreatic pseudo cyst.

\section{Case Presentation}

A 21-year-old male, presented to the emergency with a chief complaint of sudden onset of severe epigastric pain, nausea and vomiting of one day duration. The patient was on VPA $600 \mathrm{mg}$ daily prescribed for primary generalized seizure disorder he had for the last 13 months. There was no history of alcohol intake, gall stone disease, hyperlipidemia, abdominal surgery/trauma or any metabolic disturbances. Apart from epigastric tenderness and guarding, all other physical findings were normal. Laboratory findings were unremarkable except for raised serum amylase level (377 U/L). Severe epigastric pain and raised amylase level suspicion of AP was confirmed by contrast enhanced computerized tomography (CECT) which revealed features suggestive of acute necrotizing pancreatitis with parenchymal necrosis $>30 \%$ (Fig. 1). Considering VPA as a culprit for causing acute pancreatitis it was stopped and replaced with another anti epileptic drug phenytoin. With persistent high grade fever and raised serum amylase level (515 U/L) the patient was transferred from surgical ward to the Intensive Care Unit (ICU) for further management. On admission the patient was anxious, tachypnoeic, tachycardic and febrile with normal BP. Systemic examination revealed diminished air entry on bilateral lung bases, mild abdominal distension, epigastric tenderness, guarding and diminished bowel sounds. Lab investigation showed leukocytosis and deranged renal function test (raised creatinine and urea level) with respiratory alkalosis noted on arterial blood gas analysis.

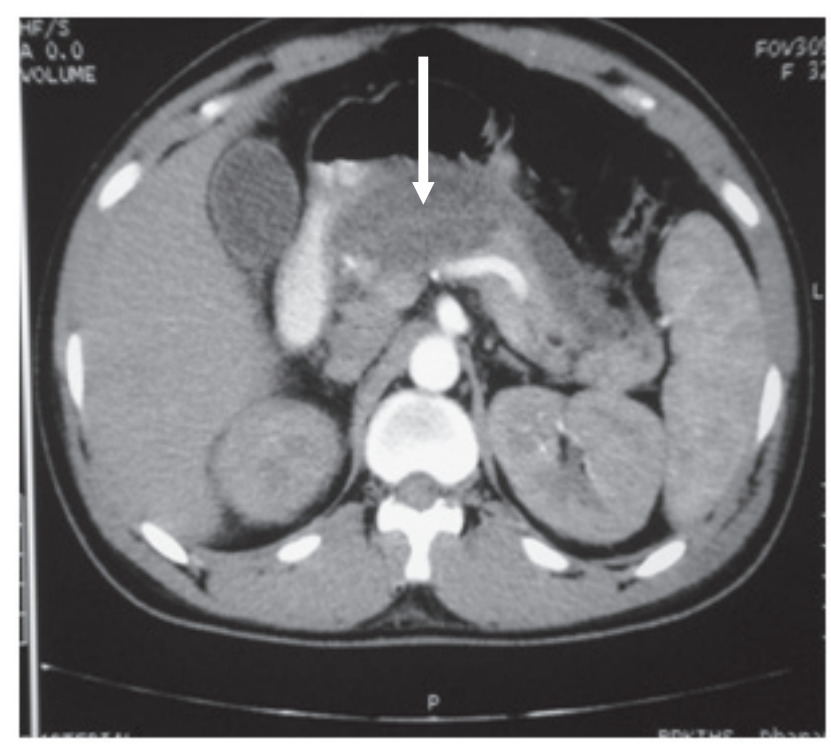

Figure. 1 CT Section showing Necrotizing Pancreatitis

Scoring on the APACHE II scale was 13. Patient was managed conservatively with intravenous fluids, analgesics and intravenous Meropenam as prophylactic antibiotic. General condition and the biochemical parameters improved and the patient was discharged on the 8th day of ICU stay. But two weeks later, he presented with mild abdominal pain, nausea, vomiting and a palpable epigastric mass which on repeat $\mathrm{CT}$ depicted as a pancreatic pseudocyst. The patient underwent an elective open necrosectomy with cystogastrostomy and cholecystectomy. Postoperative course was uneventful and was discharged 10 days later in fair general condition.

\section{Discussion}

Multiple etiological factors have been implicated in the causation of acute pancreatitis. More than eighty percent of incidence of acute pancreatitis is related to gall stone disease and alcohol consumption. Drugs, hypertriglyceridemia and hypercalcemia are other etiological factors for AP. However, the most important differential diagnoses that could be thought of besides acute pancreatitis are perforated gastric/duodenal ulcer, biliary colic, intestinal obstruction, mesenteric ischaemia, aortic dissection and acute coronary syndromes ${ }^{2}$. With detailed history, examination and appropriate investigation the aforementioned differential diagnosis were ruled out.

All guidelines agree that the diagnosis of AP should include at least two of the following features: 1 ) acute abdominal pain characteristic for pancreatitis, 2) elevated levels of serum amylase and/or lipase $\geq 3$ times the upper normal limit, 3) characteristic findings on computed tomography scan or related radiographic techniques..$^{2-5}$ Although lipase is considered to be more specific ${ }^{6}$ and sensitive ${ }^{7}$ (especially in alcohol induced pancreatitis) we could not perform serum lipase in our patient due to unavailability of the test. Certain guidelines ${ }^{4}$ recommend determining lipase level in acute pancreatitis patients with delayed presentation as it has longer half life than amylase. Nevertheless, as our patient did not have history of alcohol consumption and presented early serum amylase level measurement could be justified.

Infectious complications are the main causes of morbidity and mortality of severe acute pancreatitis patients in the later stage of the disease. ${ }^{8}$ In severe acute pancreatitis, the rate of pancreatic necrosis infection is 30 to $50 \%$ and correlates with both the extent of necrosis and the duration of pancreatitis. ${ }^{9}$ A meta-analysis observed a reduction in the length of hospital stay in severe acute pancreatitis patients receiving prophylactic antibiotics, but could not detect any effect on the rate of infectious complications or mortality. ${ }^{10}$ However, the Japanese working group ${ }^{10}$ and the International Association of Pancreatology ${ }^{11}$ recommend antibiotic prophylaxis in SAP patients to reduce the rate of necrosis infection. When choosing the appropriate antibiotic, it is important not only to think about the spectrum of organisms that it covers but also about adequate pancreatic tissue penetration. ${ }^{12}$ It has 
been shown that carbapenems fulfil both the purposes.

With no clear etiology defined as an absence of a history of alcohol intake, absence of imaging demonstrating gallstones, and a normal triglyceride level, it is reasonable to consider a drug as the cause of acute pancreatitis. ${ }^{13}$ Furthermore, our patient had no other contributing factors except for the use of VPA that might have led to the development of acute pancreatitis.

In a population-based retrospective analysis, the incidence of drug-induced pancreatitis was found to be $5.3 \%$, the third most frequent cause of acute pancreatitis after gallstones and alcohol. VPA is one of those Class I drugs with the most evidence of causing acute pancreatitis. The association of acute pancreatitis and VPA therapy was first described in 1979 by Bataladen et al ${ }^{14}$ and Camfield et al. ${ }^{15}$ Since then more than a hundred cases of VPA induced pancreatitis have been reported and reviewed in various literatures all around the world. But the actual number of cases could be more as they are either not reported or not identified.

Likewise, only a very few cases of VPA associated necrotizing pancreatitis have been reported. Sinclair notified 2 such cases in 5 and 8 years old child in her 11 case series of VPA associated pancreatitis in pediatric age group. ${ }^{16}$ Younger age group and first year of initiation of treatment have been determined as risk factors for VPA associated pancreatitis. In most patients, early discontinuation of the drug resulted in successful resolution of the symptoms, as was in our case. Furthermore, dose, duration of treatment, serum valproic acid levels, generic preparation, and the presence of concomitant antiepileptic drugs have not shown any implication in causing pancreatitis. ${ }^{15-17}$ Altered renal function test with raised serum creatinine of $6.4 \mathrm{mg} /$ $\mathrm{dl}$ and urea of $126 \mathrm{mg} / \mathrm{dl}$ in our patient with previously normal renal function could be due to contrast used for CECT - contrast induced nephropathy, although AP could also be attributable.

The mechanism of association of VPA and pancreatitis is not clearly understood. Although it has been suggested as 'idiosyncratic', depletion of the free radical scavengers superoxide dismutase, catalase, and glutathione peroxidase with resulting excess of free radicals leading to capillary endothelial injury and tissue damage has been proposed. ${ }^{18}$

\section{Conclusion}

Acute pancreatitis is a serious condition with significant morbidity and mortality despite optimal treatment. Today, valproic acid is widely used for multiple disease conditions. Starting from 1979 till 2010, 107 cases of valproic acid induced acute pancreatitis or acute necrotizing pancreatitis have been reported. Unfortunately, it is found to be underreported or undetected. Hence, increasing frequency of valproic acid associated pancreatitis demands reconsideration regarding its routine use in clinical practice.

\section{References}

1. Santos BL, Fernandes RMF, Neves FF. Arq Neuropsiquiatr 2010;68:135-36.

2. Banks PA, Freeman ML. Practice guidelines in acute pancreatitis. Am J Gastroenterol 2006;101:2379-2400.

3. American Gastroenterological Association. AGA institute medical position statement on acute pancreatitis. Gastroenterology 2007;132:2019-21.

4. UK Working Party on Acute Pancreatitis. UK guidelines for the management of acute pancreatitis. Gut 2005;54:1-9.

5. JPN. JPN guidelines for the management of acute pancreatitis. J Hepatobiliary Pancreat Surg 2006;13:10-67.

6. Sternby B, O Brien JF, Zinsmeister AR, DiMagno EP. What is the best biochemical test to diagnose acute pancreatitis? A prospective clinical study. Mayo Clin Proc 1996;71:1138-44.

7. Gumaste V, Dave P, Sereny G. Serum lipase: a better test to diagnose acute alcoholic pancreatitis. Am J Med 1992;92:239-42.

8. Carnovale A, Rabitti PG, Manes G, Esposito P, Pacelli L, Uomo G. Mortality in acute pancreatitis: is it an early or a late event? JOP 2005;6:438-44.

9. Frossard JL, Steer ML, Pastor CM. Acute pancreatitis. Lancet 2008;371:143-52.

10. Bai Y, Gao J, Zou DW, Li ZS. Prophylactic antibiotics cannot reduce infected pancreatic necrosis and mortality in acute necrotizing pancreatitis: evidence from a meta-analysis of randomized controlled trial. Am J Gastroenterol 2008;103:104-10.

11. Uhl W, Warshaw A, Imrie C, Bassi C, McKay CJ, Lankisch PG et al. IAP Guidelines for the Surgical Management of Acute Pancreatitis. Pancreatology 2002;2:565-73.

12. Buchler $M$, Malfertheiner $P$, Friess $H$. Human pancreatic tissue concentration of bactericidal antibiotics. Gastroenterology 1992;103:1902-8.

13. Tenner S. Drug-Induced Acute Pancreatitis: Underdiagnosis and Overdiagnosis. Dig Dis Sci 2010;55:2706-8.

14. Bataladen PB, Van Dyne BJ, Cloyd J. Pancreatitis associated with valproic acid therapy. Pediatrics 1979;64:520-2.

15. Camfield PR, Bagnell P, Camfield CS. Pancreatitis due to valproate acid. Lancet 1979;1(8127):1198-9.

16. Sinclair DB, Berg M, Breault R. Valproic acid-induced pancreatitis in childhood epilepsy: case series and review. J Child Neurol 2004;19:498-502.

17. Gerstner T, Busing D, Bell N, Longin E, Kasper JM, Klostermann W, et al. Valproic acid-induced pancreatitis: 16 new cases and a review of the literature. J Gastroenterol 2007;42:39-48.

18. Pippenger $C E$, Xianzhong $M$, Rothner $A D$. Free radical scavenging enzyme activity profiles in risk assessment of idiosyncratic drug reactions: probable mechanism of valproate induced pancreatitis and hepatotoxicity. In Levy RH, Penry JK, editors. Idiosyncratic reactions to valproate: clinical risk patterns and mechanism of toxicity. New York: Raven Press; 1991:75-88. 